\title{
Efficient Experimental Designs for Hyperparameter Estimation: Learning When Effect-Sizes are Large
}

\author{
by \\ Qing Liu \\ Department of Statistics \\ Ohio State University \\ qliu@stat.ohio-state.edu \\ Angela Dean \\ Department of Statistics \\ Ohio State University \\ amd@stat.ohio-state.edu \\ David Bakken \\ Harris Interactive \\ DBakken@HarrisInteractive.com
}

Greg M. Allenby

Fisher College of Business

Ohio State University

allenby.1@,osu.edu

April, 2007 


\title{
Efficient Experimental Designs for Hyperparameter Estimation: Learning When Effect-Sizes are Large
}

\begin{abstract}
$\underline{\text { Abstract }}$
Research in marketing, and business in general, involves understanding when effect-sizes are expected to be large and when they are expected to be small. Understanding the contexts in which consumers are sensitive to offers and variables such as price, is an important aspect of merchandising, selling and promotion. In this paper, we propose efficient methods of learning about contextual factors that influence consumer preference and sensitivities within the context of a hierarchical Bayes model. A design criterion is developed for hierarchical linear models, and validated in a study of the "level effect" in conjoint analysis using a national sample of respondents. Extensions to other model structures are discussed.
\end{abstract}




\title{
Efficient Experimental Designs for Hyperparameter Estimation: Learning When Effect-Sizes are Large
}

\author{
1. Introduction \\ Consumer judgment, preferences and sensitivities are context-dependent. Many studies in \\ the behavioral decision theory literature document the effects of variables such as the range \\ (Janiszewski and Lichtenstein, 1999), similarity (Tversky, 1977), and order (Kahn, Moore, and \\ Glazer, 1987) of stimuli that indicate consumer preferences are not stable, but depend on the \\ purchase and consumption environment. These results have important implications for the timing \\ of offerings, retail assortment choice, and other merchandising decisions. \\ The ability to calibrate the influence of contextual variables on consumer behavior requires \\ models that describe the variation of model parameters such as price sensitivity coefficients. Such \\ models are often written hierarchically, with a first layer of the model describing respondent reaction \\ to stimuli, and a second layer describing contextual factors that drive the magnitude of effect sizes. \\ The parameters in this second layer are commonly referred to as hyperparameters because they \\ describe the characteristics of the parameters in the first layer. \\ In this paper, we describe efficient designs for the learning of hyperparameters in \\ hierarchical linear models and illustrate their use. A design criterion is proposed that can be used for \\ a range of hierarchical models and, as a special case, the criterion includes the D-criterion for \\ traditional fixed-effects linear models. We illustrate the design performance through a study of the \\ level effect in conjoint analysis, where part-worth estimates have been shown to be positively \\ associated with the number of attribute-levels present in the study. This form of context \\ dependence has implications for predicting preferences in environments characterized by varying \\ levels of assortment across different retailers. Data from a national survey were used to validate our \\ methodology.
}


The remainder of the paper is organized as follows: section 2 describes the optimal design criterion for the estimation of hyperparameters in a hierarchical linear model. Section 3 introduces the theory and parameterization for studying the level effect in conjoint analysis. Designs are constructed and compared in section 4 , and section 5 reports empirical results from a national webbased survey. Concluding remarks are offered in section 6.

\section{Design Criterion for Hyperparameters}

Consider a consumer survey in which respondent $\mathrm{i}(\mathrm{i}=1, \ldots, \mathrm{n})$ is presented with a set of $\mathrm{m}_{\mathrm{i}}$ profiles (stimuli). The stimuli are constructed with information on various levels of marketing variables, such as price, product attributes or possibly aspects of advertisements. The levels of the marketing variables are reflected in the $\mathrm{m}_{\mathrm{i}} \times \mathrm{p}$ model matrix, $\mathrm{X}_{\mathrm{i}}$. The responses of consumer $\mathrm{i}$ to the set of stimuli are represented by the vector $y_{i}$ of length $m_{i}$. The effects of the various levels of marketing variables on respondent i's responses are captured by the $\mathrm{p}$ elements of vector $\beta_{\mathrm{i}}$, which are assumed to be random effects distributed according to a multivariate normal distribution with

mean $Z_{i} \theta$ and variance-covariance matrix $\Lambda(p \times p) . Z_{i}$ is a matrix $(p \times q)$ of covariates, such as household income, age or other contextual variables that characterize the purchasing environment. $\theta$ is a parameter vector of length $\mathrm{q}$. Thus, the hierarchical linear model is of the following form:

$$
\begin{aligned}
& y_{i} \mid \beta_{i}, \sigma^{2}=\mathrm{X}_{i} \beta_{i}+\varepsilon_{i} \\
& \beta_{i} \mid \theta, \Lambda=\mathrm{Z}_{i} \theta+\delta_{i}
\end{aligned}
$$


The error vector, $\varepsilon_{\mathrm{i}}$ of length $\mathrm{m}_{\mathrm{i}}$ in the first level of the hierarchy captures consumer i's response variability to the set of stimuli, and it is assumed to have a Multivariate Normal distribution with mean vector 0 of length $\mathrm{m}_{\mathrm{i}}$ and variance-covariance matrix $\sigma^{2} \mathrm{I}_{m_{i}}$. The error vector $\delta_{\mathrm{i}}$ of length $\mathrm{p}$ in the second level of the hierarchy captures the dispersion of the individual-level effects $\beta_{\mathrm{i}}$ and is assumed to be Multivariate Normal with mean vector 0 and variance-covariance matrix $\Lambda$ of size $\mathrm{p} \times \mathrm{p}$. When the prior knowledge is weak, the following proper but diffuse priors are usually assumed for $\theta, \Lambda$ and $\sigma^{2}$ (see Gamerman 1997; Rossi, Allenby and McCulloch 2005). These are replaced by more informative priors when prior knowledge is available.

$$
\begin{aligned}
& \theta \sim \operatorname{Normal}\left(0,100 \mathrm{I}_{\mathrm{p}}\right) \\
& \Lambda \sim \text { Inverted Wishart }\left(\mathrm{v}_{0}=\mathrm{p}+3, \mathrm{~V}_{0}=\mathrm{v}_{0} \mathrm{I}_{\mathrm{p}}\right) \\
& \sigma^{2} \sim \text { Inverse Gamma }(3 / 2,1 / 2)
\end{aligned}
$$

Many researchers have investigated efficient experimental designs for the estimation of the individual-level random effects $\beta_{i}$ under hierarchical models (See, for example, Arora and Huber, 2001; Sándor and Wedel, 2001, 2005; Kessels et al., 2006). While it is important to have accurate information on $\beta_{\mathrm{i}}$ when the focus is on individual-level customization of products, accurate estimation of hyperparameters $\theta$ and/or $\Lambda$ is important in other situations. This occurs when individual-level targeting is not possible, when the mean effects of marketing variables on a population of consumers are of interest, or when predictions of consumer preferences in a new target population are required.

Pragmatic approaches to finding efficient designs have been proposed for the estimation of hyperparameters under a hierarchical nonlinear model. For example, the swapping, relabeling and cycling heuristic by Sándor and Wedel (2002); the linearization approach by Mentré et al. (1997); the stochastic gradient search by Tod et al. (1998), and the "MCMC nested within Monte Carlo" 
approach by Han and Chaloner (2004). No contextual variables are included in the hierarchical models used in these papers, that is, $Z_{\mathrm{i}}$ equals the identity matrix.

Under a hierarchical linear model with independent, homoscedastically distributed random effects (i.e., $\Lambda=\lambda^{2} \mathrm{I}$ ), Lenk et al. (1996) analytically investigate, in the survey setting, the tradeoff between the number of subjects and the number of questions per subject under a cost constraint and an orthogonal design structure. Contextual variables are included in the model but $\mathrm{Z}_{\mathrm{i}}$ is assumed given, that is, $Z_{\mathrm{i}}$ is not under the control of the experimenter. Liu et al. (2007) study optimal designs for the estimation of hyperparameters under a hierarchical linear model for both the situation of independent random effects and that of correlated random effects. A brief discussion is given for the situation when both the stimuli $\left(\mathrm{X}_{\mathrm{i}}\right)$ and contextual variables $\left(\mathrm{Z}_{\mathrm{i}}\right)$ can be controlled by the experimenter.

In this paper, we build on Liu et al. (2007) and explore efficient experimental designs expressed in terms of the stimuli $\left(\mathrm{X}_{\mathrm{i}}\right)$ and contextual variables $\left(\mathrm{Z}_{\mathrm{i}}\right)$. Contextual variables are broadly defined to include descriptors of individuals (e.g., demographics), variables describing the purchase and/or consumption environment (e.g., merchandising assortment, social affects), and variables that come from the intersection of these factors (e.g., needs). Learning about the influence of these variables enables marketers to identify who is most likely to prefer an offering, and the conditions associated with strong preference.

The presence of context-dependent preferences also has implications for data-collection, including selection of respondents using demographic quotas, wording of questions and presentation of stimuli. The elicitation of consumer preferences involves a complicated process of encoding, memory activation, and reporting that can be affected by the same variables that are used to explain contextual variation. Thus, the design of experiments to understand the variation of effect-sizes requires methods that optimize the gain of information with respect to the specific 
stimuli, as well as variables that describe the context of the stimuli. Traditional statistical design criteria focus only on the former. We next introduce such a design criterion and illustrate its use in Section 4.

\section{Design Optimality Criterion for Hyperparameter Estimation}

We consider the situation when the primary interest is on the accurate estimation of the context effect vector $\theta$, or a function $g(\theta)$ of vector $\theta$, while the response error variance $\sigma^{2}$ and the random effects variance-covariance matrix $\Lambda$ are treated as nuisance parameters. Equations (1) and (2) can be combined to obtain

$$
y_{i} \mid \theta, \Lambda, \sigma^{2} \sim \mathrm{N}_{m_{i}}\left(\mathrm{X}_{i} \mathrm{Z}_{i} \theta, \quad \Sigma_{i}=\sigma^{2} \mathrm{I}_{m_{i}}+\mathrm{X}_{i} \Lambda \mathrm{X}_{i}^{\prime}\right) \text {, }
$$

where $\mathrm{N}_{m_{i}}$ denotes multivariate normal distribution with dimension $\mathrm{m}_{\mathrm{i}}$, with priors (3) to (5) assumed for $\theta, \Lambda$ and $\sigma^{2}$.

In Liu et al. (2007), it is shown that, for the estimation of the context effect vector $\theta$, an optimal design that maximizes the expected gain in Shannon information (see Chaloner and Verdinelli, 1995, page 277) is a design with matrices $\left\{\mathrm{X}_{\mathrm{i}}\right\}$ and $\left\{\mathrm{Z}_{\mathrm{i}}\right\}$ (if $\mathrm{Z}_{\mathrm{i}}$ can be controlled by the experimenter), $i=1, \ldots, n$, that maximizes

$$
\psi=\int\left\{\log \left|\sum_{i=1}^{n} \mathrm{Z}_{i}^{\prime} \mathrm{X}_{i}^{\prime}\left(\sigma^{2} \mathrm{I}_{m_{i}}+\mathrm{X}_{i} \Lambda \mathrm{X}_{i}^{\prime}\right)^{-1} \mathrm{X}_{i} \mathrm{Z}_{i}\right|\right\} p(\Lambda) p\left(\sigma^{2}\right) d \Lambda d \sigma^{2}
$$

where $\mathrm{p}(\Lambda)$ and $\mathrm{p}\left(\sigma^{2}\right)$ denote the probability density functions of the prior distributions of $\Lambda$ and $\sigma^{2}$. We note that if $Z_{i}=I$ (i.e., no contextual variables), $X_{i}=X$ (i.e., same stimuli for all respondents), $\Lambda=0$ (i.e., no heterogeneity), and $\sigma^{2}$ is known, then the $\psi$-criterion is equivalent to the classical Dcriterion for the estimation of fixed effects. The $\psi$-criterion in equation (7) can therefore be regarded as a generalization of the classical fixed-effects D-criterion. 
In certain situations when all respondents receive the same stimuli $\left(\mathrm{X}_{\mathrm{i}}=\mathrm{X}\right), \mathrm{X}$ can be determined independently of $\left\{Z_{i}\right\}$ in an optimal design under the $\psi$ criterion. For example, in a survey study, when the same questionnaire is given to all respondents, the optimal construction of the questionnaire is often independent of the optimal sampling of the respondents on the basis of demographic information such as age or income. However, in other situations when respondents receive customized stimuli or when the construction of the stimuli is linked to the contextual variables, optimal $\left\{\mathrm{X}_{\mathrm{i}}\right\}$ and $\left\{\mathrm{Z}_{\mathrm{i}}\right\}$ need to be determined jointly.

In this paper, we extend Liu et al. (2007) and focus on the estimation of a function $g(\theta)$ of hyperparameter vector $\theta$. Appendix A shows in detail that an optimal design for the estimation of $\mathrm{g}(\theta)$ is a design with matrices $\left\{\mathrm{X}_{\mathrm{i}}\right\}$ and $\left\{\mathrm{Z}_{\mathrm{i}}\right\}, \mathrm{i}=1, \ldots, \mathrm{n}$, that maximizes

$$
\tilde{\psi}=\int\left\{-\log \left|\left(\frac{\partial g(\theta)}{\partial \theta}\right)^{\prime}\left(\sum_{i=1}^{n} \mathrm{Z}_{i}^{\prime} \mathrm{X}_{i}^{\prime}\left(\sigma^{2} \mathrm{I}_{m_{i}}+\mathrm{X}_{i} \Lambda \mathrm{X}_{i}^{\prime}\right)^{-1} \mathrm{X}_{i} \mathrm{Z}_{i}\right)^{-1}\left(\frac{\partial g(\theta)}{\partial \theta}\right)\right|\right\} p(\theta) p(\Lambda) p\left(\sigma^{2}\right) d \theta d \Lambda d \sigma^{2}
$$

where $\mathrm{p}(\theta)$ is the probability density function of the prior distribution of $\theta$.

We use the $\tilde{\psi}$-criterion to select the survey designs in Section 4 in the context of the level effect in conjoint analysis where $\mathrm{X}_{\mathrm{i}}$ is linked to $\mathrm{Z}_{\mathrm{i}}$. The next section introduces the level effect, and proposes a hierarchical linear model that captures the level effect as one of the hyperparameters.

\section{The Level Effect in Conjoint Analysis}

Currim, Weinberg and Wittink (1981) first observed that part-worth estimates in a conjoint study increase with the addition of intermediate attribute levels. This phenomenon was named the number-of-attribute levels (NOL) effect, also known as the level effect. Since then, the level effect has been observed across different measurement scales, various data collection methods and estimation techniques (see Wittink et al., 1982, 1990, 1997; Steenkamp and Wittink, 1994; and Creyer and Ross, 1988). 
Possible explanations of the level effect have been proposed in the literature. Currim, Weinberg and Wittink (1981) find that the ordinal properties of ranking and rating measures contribute to the occurrence of the level effect. Wittink et al. (1992), Steenkamp and Wittink (1994), Verlegh, Schifferstein and Wittink (2002) find that certain experimental design methods such as the utility-balance approach in ACA (Adaptive Conjoint Analysis), and small sampling errors seem to reduce the magnitude of the level effect. Steenkamp and Wittink (1994) also investigate the attention-based explanation which states that the respondents' attention to an attribute may increase with the addition of attribute levels. However, only minimal support of the attention-based explanation has been found in empirical studies. Most recently, Verlegh, Schifferstein and Wittink (2002) suggest that the level effect is most likely due to respondents' tendencies to uniformly distribute their responses over the corresponding continuum of the measurement scale.

The existence of the level effect suggests that consumer preference varies with context. Specifically, it implies that, in the market place, consumer preference sensitivity to a product attribute is affected by the variety of attribute levels displayed, which varies from store to store. Such a finding is consistent with a large body of consumer behavior literature (e.g., Huber, Payne and Puto, 1982; Lynch, Chakravarti and Mitra, 1991; Simonson and Tversky, 1992) describing the influence of contextual effects in choice. Standard conjoint analysis models in marketing research do not adjust for contextual influences in part-worth estimation and, consequently, can not predict consumer buying behavior well in different contexts. In this section, we attempt to model the level effect by incorporating two ideas from the psychology literature - the Range-Frequency theory of Parducci $(1965,1974,1982)$ and Krumhansl's (1978) Distance-Density model. We first specify the model for a single attribute, and then express the model in its general form. 


\section{The Model: Single-Attribute Case}

Parducci (1965) proposes a theory on how evaluation is influenced by two factors - the range and frequency of levels of an attribute. His theory posits that preferences reflect a compromise between these factors, where range is defined in terms of a linear mapping of attribute-levels to a measure of value, and frequency is defined in terms of an ordinal mapping to value. To illustrate, suppose respondents are presented with five possible car prices:

$$
\$ 20,000 \quad \$ 21,000 \quad \$ 26,000 \quad \$ 28,000 \quad \$ 30,000
$$

Evaluations based on the principle of range leads to values of:

$\begin{array}{lllll}10 & 9 & 4 & 2 & 0\end{array}$

and evaluations based on frequency result in values of:

$\begin{array}{lllll}10 & 7.5 & 5 & 2.5 & 0\end{array}$

Cooke et al. (2004) formalize Parducci's range-frequency theory into the following model:

$$
\begin{aligned}
& R_{g}=\left(S_{g}-S_{\text {min }}\right) /\left(S_{\text {max }}-S_{\text {min }}\right) \\
& F_{g}=(\operatorname{Rank}(g)-1) /(N-1) \\
& J_{g}=\omega R_{g}+(1-\omega) F_{g}
\end{aligned}
$$

where $J_{g}$ denotes a normalized value of attribute-level $g$ as a weighted average of the range $\left(R_{g}\right)$ and frequency $\left(\mathrm{F}_{\mathrm{g}}\right)$ effects with $0 \leq \omega \leq 1$. Cooke et al. (2004) transform this normalized value to an actual value by the equation:

$$
A_{g}=b+m J_{g}
$$

where $\mathrm{b}$ is the respondent's perceived value of the lowest-level of the attribute, and $\mathrm{m}$ is the "perceived distance" between the lowest and highest attribute-levels.

We extend the model proposed by Cook et al. (2004), (9) to (12), by modeling $m$ according to the Distance-Density model in Krumhansl (1978) which states that "...two points in a relatively 
dense region of a stimulus space would have a smaller similarity measure than two points of equal interpoint distance but located in a less dense region of the space...". Specifically, we assume $m$ is a linear function of the number of attribute levels $L$ :

$$
m=\kappa+v L
$$

and the final form of the model is:

$$
A_{g}=b+\kappa F_{g}+v L F_{g}+\kappa \omega\left(R_{g}-F_{g}\right)+v \omega L\left(R_{g}-F_{g}\right)+\delta_{g}
$$

where $\delta_{g}$ is an error term distributed according to a $\mathrm{N}\left(0, \lambda^{2}\right)$ distribution that allows for respondentspecific deviation from the assumed structure.

The parameter $v$ in equation (14) measures the level effect in conjoint analysis. To estimate $v$, it is required that the conjoint study contains multiple parts which have different numbers of attribute levels $L$. The difference $R_{g}-F_{g}$ in equation (14) represents how far away the relative range is from the relative rank and provides a measure of the skewness of the distribution of the attribute levels. If an attribute has levels packed on the lower end, for example, (10, 15, 20, 30, 35, 40, 70, 90, 100), then we will observe prevailingly $R_{g}-F_{g}<0$. Similarly, if an attribute has levels that are packed up on the higher end, we will have prevailingly $R_{g}-F_{g}>0$. If an attribute has equally-spaced levels, or when an attribute has only 2 levels, then $R_{g}-F_{g}=0$ for all $g$. So, for $\omega$ in equation (14) to be estimable, we need to have a part of the study in which the attribute has more than two levels and the levels are not equally spaced.

\section{The Model: General Expression in Matrix Form}

We now express the model in matrix form for the general case of multiple respondents rating multiattribute products in a conjoint study. The model takes the form of a hierarchical linear model:

$$
y_{i, s} \mid \beta_{i, s}, \sigma^{2} \sim \operatorname{Normal}\left(\mathrm{X}_{i, s} \beta_{i, s}, \sigma^{2} \mathrm{I}\right)
$$


where $s$ indexes the parts of the conjoint study $(s=1,2, \ldots, S)$ with possibly different numbers of attribute levels. $\mathrm{y}_{\mathrm{i}, \mathrm{s}}$ is the vector of profile ratings from respondent $i(i=1, \ldots, \mathrm{n})$ in part $s$ of the study, $\beta_{\mathrm{i}, \mathrm{s}}$ is the vector of attribute-level part-worths, and $\mathrm{X}_{\mathrm{i}, \mathrm{s}}$ is the model matrix representing the stimuli presented to respondent $i$ in part $s$ of the study. If all respondents in part $s$ of the study are asked to rate the same set of stimuli, then the model matrix is the same across all respondents in that part of the study, that is, $\mathrm{X}_{\mathrm{i}, \mathrm{s}}=\mathrm{X}_{\mathrm{s}}$. Note that (15) for a given part $s$ of the study is the same as (1), the first level of the hierarchical model introduced in Section 1.

In the second level of the hierarchical model, the part-worths $\beta_{\mathrm{i}, \mathrm{s}}$ are modeled according to the Range-Frequency/Distance-Density theory as formalized in Equation (14). The expression of the second level of the hierarchical model is:

$$
\beta_{i, s} \mid \theta, \Lambda_{s} \sim \operatorname{Normal}\left(\mathrm{Z}_{s} \theta, \Lambda_{s}\right)
$$

The hyperparameter vector $\theta$ is defined as

$$
\theta^{\prime}=\left(\mu, \theta_{1}^{\prime}, \ldots, \theta_{\tau}^{\prime}, \ldots, \theta_{\mathrm{T}}^{\prime}\right)
$$

where $\tau$ indicates the $\tau^{\text {th }}$ attribute $(\tau=1, \ldots, T)$. When attribute $\tau$ is monotonic with different numbers of levels across the multiple parts of the study, $\theta_{\tau}^{\prime}=\left(\kappa_{\tau}, v_{\tau}, \kappa_{\tau} \omega_{\tau}, v_{\tau} \omega_{\tau}\right)$, where the set of $\left(\kappa_{\tau}, \cup_{\tau}, \omega_{\tau}\right)$ corresponds to the $(\kappa, v, \omega)$ in (14) for the attribute $\tau$. If the monotonic attribute $\tau$ has the same number of levels across the multiple parts of the study, $\mathbf{v}_{\tau}$ is not estimable and therefore $\theta_{\tau}{ }^{\prime}$ $=\left(\kappa_{\tau}, \kappa_{\tau} \omega_{\tau}\right)$. Similarly, if the attribute levels in attribute $\tau$ are equally-spaced or only contain the two extreme levels in all parts of the study, $\omega_{\tau}$ is not estimable and therefore $\theta_{\tau}^{\prime}=\left(\kappa_{\tau}, \cup_{\tau}\right)$. Finally, for non-monotonic attributes such as brand name or color, the vector $\theta_{\tau}$ simply contains the means of the individual-level effects over the respondents.

The covariate matrix $Z_{s}$ in (16) is of a block diagonal structure: 


$$
\mathrm{Z}_{s}=\left(\begin{array}{cccccc}
1 & 0 & \cdots & 0 & \cdots & 0 \\
0 & \mathrm{Z}_{1, s} & \cdots & 0 & \cdots & 0 \\
\vdots & \vdots & \ddots & \vdots & & \vdots \\
0 & 0 & \cdots & \mathrm{Z}_{\tau, s} & \cdots & 0 \\
\vdots & \vdots & & \vdots & \ddots & \vdots \\
0 & 0 & \cdots & \cdots & \cdots & \mathrm{Z}_{T, s}
\end{array}\right)
$$

$Z_{\tau, s}=1$ when attribute $\tau$ is non-monotonic. $Z_{\tau, s}$ is a matrix that consists of $F, L_{\tau, s} F,(R-F)$ and $L_{\tau, s}(R-F)$ on the various levels of attribute $\tau$, as required by Equation (14), when attribute $\tau$ is a monotonic with different numbers of levels across the multiple parts of the study. $Z_{\tau, s}$ consists only of $F$ and $R-F$ on the various levels of attribute $\tau$ if the monotonic attribute $\tau$ has the same number of levels across studies, and $Z_{\tau, \mathrm{s}}$ consists only of $F$ on various levels of the attribute $\tau$ if the attribute levels in attribute $\tau$ are equally-spaced or only contain the two extreme levels in all parts of the study.

Appendix B illustrates the model matrices $\mathrm{X}_{\mathrm{i}, \mathrm{s}}$ and covariate matrices $\mathrm{Z}_{\mathrm{s}}$ in our hierarchical model (15) and (16) for an investigation of the level effect of credit card interest rate (APR). These design matrices were selected using the $\tilde{\psi}$-criterion in equation (8) that accounts for the dependence of $\mathrm{Z}_{\mathrm{i}}$ on $\mathrm{X}_{\mathrm{i}, \mathrm{s}}$ due to the level effect. Next we illustrate the importance of accounting for this dependence when estimating contextual factors that influence consumer valuation of product offerings.

\section{Survey Designs for Studying the Level Effect}

A survey study was conducted to evaluate the effectiveness of the proposed model, through Harris Interactive, a global marketing research firm. Credit card products were used as stimuli in the survey. Measurement of the level effect in credit-card preferences requires that the study contains multiple parts where the number of attribute levels varies. The attributes used in the survey studies were the APR (interest rate) of the card, the card provider and the reward program. Part 1 of the 
study involved the two extreme levels $(8.99 \%$ and $17.99 \%)$ of APR in the study, part 2 involved four levels of APR with the addition of two intermediate levels, and part 3 involved three levels of APR with the addition of one intermediate level. The numbers of levels of the other two attributes, card provider and reward program, remained the same across the three parts of the study. In particular, there were two card providers -- Capital One vs. Citibank, and three levels of rewards - none, cash reward and travel reward. Data from parts 1 and 2 of the study were used to estimate the model parameters, and part 3 was reserved for holdout predictive testing. Each respondent in the survey was asked to evaluate 12 credit card products on a 0 to 10 rating scale, with 0 being least likely to apply and 10 being most likely to apply.

The vector $\theta$ of the hierarchical model in (17) is

$$
\theta=\left(\mu, \kappa_{\mathrm{A}}, \mathrm{v}_{\mathrm{A}}, \kappa_{\mathrm{A}} \omega_{\mathrm{A}}, \mathrm{v}_{\mathrm{A}} \omega_{\mathrm{A}}, \theta_{\mathrm{P}}, \theta_{\mathrm{C}}, \theta_{\mathrm{T}}\right)^{\prime},
$$

where the $\left(\kappa_{\mathrm{A}}, \mathrm{v}_{\mathrm{A}}, \omega_{\mathrm{A}}\right)$ reflect the $(\kappa, \mathrm{v}, \omega)$ in (14) on the APR attribute, $\theta_{\mathrm{P}}$ is the mean contrast between the two card providers, $\theta_{\mathrm{C}}$ is the mean contrast between cash reward and no reward, and $\theta_{\mathrm{T}}$ is the mean contrast between travel reward and no reward. The vector $\theta$ is a nonlinear function of $\theta^{*}$ which contains the unique parameters to be estimated in (19), that is,

$$
\theta^{*}=\left(\mu, \kappa_{\mathrm{A}}, v_{\mathrm{A}}, \omega_{\mathrm{A}}, \theta_{\mathrm{P}}, \theta_{\mathrm{C}}, \theta_{\mathrm{T}}\right)^{\prime} .
$$

For efficient estimation of $\theta^{*}$ through parts 1 and 2 of the study, the construction of the stimuli (which determines $\left\{\mathrm{X}_{\mathrm{i}, \mathrm{s}}\right\}$ ) and the physical values of APR (which determines $\left\{\mathrm{Z}_{\mathrm{s}}\right\}$ ) for the two parts need to be jointly determined so that $\tilde{\psi}$ in (8) is maximized. Note that $\mathrm{Z}_{1}$ in part 1 of the study was fixed since the two extreme APR levels were fixed at $8.99 \%$ and $17.99 \%$. To simplify the problem, the same set of stimuli was assumed to be presented to the respondents in the same part of the study $\left(\mathrm{X}_{\mathrm{i}, \mathrm{s}}=\mathrm{X}_{\mathrm{s}}\right)$, and a full-factorial design with twelve profiles, as shown in Table B.1 of 
Appendix B, was used in part 1 of the survey. Therefore, $\mathrm{X}_{1}$ was fixed, and the problem was simplified to the search of $\mathrm{X}_{2}$ and $\mathrm{Z}_{2}$ for part 2 of the study that maximize

$$
\begin{aligned}
\tilde{\psi}=\int\left\{-\log \left|\left(\frac{\partial g(\theta)}{\partial \theta}\right)^{\prime}\left(\sum_{s=1}^{2} \mathrm{Z}_{s}^{\prime} \mathrm{X}_{s}^{\prime}\left(\sigma_{s}^{2} \mathrm{I}_{12}+\mathrm{X}_{s} \Lambda_{s} \mathrm{X}_{s}^{\prime}\right)^{-1} \mathrm{X}_{s} \mathrm{Z}_{s}\right)^{-1}\left(\frac{\partial g(\theta)}{\partial \theta}\right)\right|\right\} \\
\times p(\theta) p\left(\Lambda_{1}\right) p\left(\Lambda_{2}\right) p\left(\sigma_{1}^{2}\right) p\left(\sigma_{2}^{2}\right) d \theta d \Lambda_{1} d \Lambda_{2} d \sigma_{1}^{2} d \sigma_{2}^{2}
\end{aligned}
$$

given an equal number of respondents in each part of the study, where

$$
\frac{\partial g(\theta)}{\partial \theta}=\frac{\partial \theta^{*}}{\partial \theta}=\left(\begin{array}{ccccccc}
1 & 0 & 0 & 0 & 0 & 0 & 0 \\
0 & 1 & 0 & 0 & 0 & 0 & 0 \\
0 & 0 & 1 & 0 & 0 & 0 & 0 \\
0 & 1 / \omega_{A} & 0 & 1 / \kappa_{A} & 0 & 0 & 0 \\
0 & 0 & 1 / \omega_{A} & 1 / v_{A} & 0 & 0 & 0 \\
0 & 0 & 0 & 0 & 1 & 0 & 0 \\
0 & 0 & 0 & 0 & 0 & 1 & 0 \\
0 & 0 & 0 & 0 & 0 & 0 & 1
\end{array}\right)
$$

Based on the credit card offers available on the market, there were five possible choices of intermediate APR levels to be included in the study -- 9.99\%, 11.99\%, 12.99\%, 14.99\%, and 15.99\%. Therefore, for part 2 of the study, there were a total of $\left(\begin{array}{l}5 \\ 2\end{array}\right)=10$ possible choices of $Z_{2}$. For each possible choice of $\mathrm{Z}_{2}$, we obtained an optimal $\mathrm{X}_{2}$ that maximizes $\tilde{\psi}$ in (21) through computer search. Then we recorded the $\widetilde{\psi}$ value for each pair of $\left(\mathrm{X}_{2}, \mathrm{Z}_{2}\right)$, and compared the $\widetilde{\psi}$ values to obtain the optimal pair of $\mathrm{X}_{2}$ and $\mathrm{Z}_{2}$. Similarly, for part 3 of the study, there were a total of 5 possible choices of $Z_{3}$. We used the same approach to obtaining the optimal $\mathrm{X}_{3}$ and $\mathrm{Z}_{3}$ by replacing the index $s=2$ with the index $s=3$ in (21). 
Table B.6 in Appendix B reports the matrices $Z_{2}$ and $Z_{3}$ in the optimal pairs $\left(X_{2}, Z_{2}\right)$ and $\left(X_{3}\right.$, $Z_{3}$ ) that were selected for parts 2 and 3 of the study, together with the fixed $Z_{1}$ for part 1 of the study. Table 1 lists the attributes and attribute-levels in the three parts of the study.

\begin{tabular}{cll}
\hline Part 1 & $\begin{array}{l}\text { APR: } \\
\text { Provider: } \\
\text { Reward: }\end{array}$ & $\begin{array}{l}(8.99 \%, 17.99 \%) \\
\text { (Capital One, Citibank) } \\
\text { (None, Cash, Travel) }\end{array}$ \\
& APR: & \\
Part 2 & Provider: & $(8.99 \%, 9.99 \%, 15.99,17.99 \%)$ \\
& Reward: & (Capital One, Citibank) \\
& (None, Cash, Travel) \\
Part 3 & APR: & $(8.99 \%, 9.99 \%, 17.99 \%)$ \\
$($ Holdout $)$ & Provider: & $\begin{array}{l}\text { (Capital One, Citibank) } \\
\text { (None, Cash, Travel) }\end{array}$ \\
& Reward: & \\
\hline
\end{tabular}

Table 1: Web-based 12-Profile Credit Card Survey Study

Simple-exchange algorithms (see Atkinson and Donev, 1992) have been used in the literature for the search of D-optimal designs. We modified a simple exchange algorithm to obtain an optimal $\mathrm{X}_{2}$ that maximizes $\widetilde{\psi}$ in (21) for given $\mathrm{X}_{1}, \mathrm{Z}_{1}$ and $\mathrm{Z}_{2}$, where the integral of $\widetilde{\psi}$ was calculated using Monte Carlo method. Based on prior knowledge, under the standardized orthogonal effects coding (see Kuhfeld, 2005) of the model matrices $\mathrm{X}_{1}$ and $\mathrm{X}_{2}$, the variance-covariance matrices $\Lambda_{1}$ and $\Lambda_{2}$ were expected to have positive off-diagonal elements, that is, the random effects were expected to be positively correlated although the actual sizes of the correlations and the variances were unknown. Following recommendations in Liu et al. (2007), the following priors were used, respectively, for $\Lambda_{1}$ and $\Lambda_{2}$, in the computer search of an optimal $\mathrm{X}_{2}$.

$$
\begin{aligned}
& \Lambda_{1} \sim \text { Inverted Wishart }\left(v_{0}=7, V_{0}=2 I_{5}+J_{5}\right), \\
& \Lambda_{2} \sim \text { Inverted Wishart }\left(v_{0}=9, V_{0}=2 I_{7}+J_{7}\right),
\end{aligned}
$$

where $\mathrm{J}$ denotes a matrix with all elements being ones. We note that, while these values of $\mathrm{V}_{0}$ were used in the search of an optimal $\mathrm{X}_{2}$, alternative values lead to the same rank ordering of candidate $\mathrm{X}_{2}$ 
matrices for a wide range of $\mathrm{V}_{0}$ values where $\mathrm{V}_{0}=a \mathrm{I}+\mathrm{bJ}$ with any positive $\mathrm{a}$ and $\mathrm{b}$ such that $0.5<a / b \leq 2$. For the rest of the parameters, non-informative priors were used as follows,

$$
\begin{aligned}
\sigma_{1}^{2}, \sigma_{2}^{2} & \sim \text { Inverse Gamma }(3 / 2,1 / 2), \\
\omega_{\mathrm{A}} & \sim \operatorname{Uniform}(0,1), \\
\theta_{-\omega_{A}}^{*} & \sim \operatorname{Normal}\left(0,100 \mathrm{I}_{6}\right),
\end{aligned}
$$

where $\theta_{-\omega_{A}}^{*}=\left(\mu, \kappa_{A}, v_{A}, \theta_{P}, \theta_{C}, \theta_{T}\right)^{\prime}$.

For purpose of comparison, in addition to an optimal $\mathrm{X}_{2}$, three other versions of designs were selected for the stimuli construction in part 2 of the study. Tables B.2 and B.3 in Appendix B list the four versions of designs and the corresponding model matrices, where Design IV denotes the optimal design obtained through computer search and Design I, II, III denote the three designs in comparison with the optimal design. Using Design I as the base design, a relative $\widetilde{\psi}$-efficiency is reported for a design with model matrix $\mathrm{X}_{2}^{\#}$, as defined by

$$
\text { Relative } \tilde{\psi} \text {-eff. }=\exp \left\{\tilde{\psi}\left(\mathrm{X}_{2}^{\#}\right)-\tilde{\psi}\left(\mathrm{X}_{2}^{b}\right)\right\} \text {, }
$$

where $X_{2}^{b}$ denotes the model matrix corresponding to Design $I$, and $\tilde{\psi}\left(X_{2}^{\#}\right)$ and $\tilde{\psi}\left(X_{2}^{b}\right)$ represent the values of $\tilde{\psi}$ in (21) corresponding to the design with model matrix $\mathrm{X}_{2}^{\#}$ and $\mathrm{X}_{2}^{b}$, respectively. As a comparison, relative D-efficiency between the two designs is also reported, as defined by

$$
\text { Relative D-eff. }=\frac{\left|\mathrm{X}_{2}^{\# \prime} \mathrm{X}_{2}^{\#}\right|}{\left|\mathrm{X}_{2}^{b^{\prime}} \mathrm{X}_{2}^{b}\right|} \text {, }
$$

where $\left|X_{2}^{\# \prime} X_{2}^{\#}\right|$ and $\left|X_{2}^{b^{\prime}} X_{2}^{b}\right|$ are values of the D-criterion function traditionally used for stimuli construction in market research under fixed-effects linear models (Kuhfeld et al. 1994). 
As shown in Table 2, Design IV has relatively the highest $\widetilde{\psi}$-efficiency and the lowest Defficiency. Design I is close to Design IV under $\widetilde{\psi}$-criterion, followed in order by Design III and Design II, with Design II being the least efficient of the four under the $\widetilde{\psi}$ criterion. On the other hand, Designs I, II and III are equally efficient under the D-criterion.

\begin{tabular}{||c|c|c|c|c||}
\hline $\begin{array}{c}\text { Relative } \\
\text { Efficiency }\end{array}$ & Design I & Design II & Design III & Design IV \\
\hline$\widetilde{\psi}$ & $100 \%$ & $95.5 \%$ & $98.4 \%$ & $100.4 \%$ \\
\hline $\mathrm{D}$ & $100 \%$ & $100 \%$ & $100 \%$ & $93.8 \%$ \\
\hline
\end{tabular}

Table 2: Relative Efficiencies of Designs for Stimuli Construction in Part 2 of the Study

Similarly, three versions of designs were selected for stimuli construction in part 3 of the study, as reported in Appendix B. Part 3 of the study was used as holdout for prediction validation. Namely, data obtained from parts 1 and 2 of the study were used to estimate the $\theta^{*}$ in (20), and the $\theta^{*}$ estimates were used to predict the mean preferences (over the respondents) in the holdout study (see Section 5).

\section{Empirical Results}

The data were collected via a national web-based survey through Harris Interactive. The following screening rules were used during the data collection: a qualified respondent needs to be 18 years of age or older, live in U.S., and have a credit card issued under his/her own name. A total of 1000 respondents participated in the study. Respondents were randomly assigned to the different parts of the study, and the presentation order of the 12 credit card profiles was also random. The data showed that, some respondents gave the same ratings to all the 12 profiles presented to them, some respondents chose one product and rated the remaining eleven products 0 , and some respondents used the scale in a reverse order (i.e., 0 was used for most likely to apply, and 10 was 
used for least likely to apply). Responses from these respondents were considered invalid and taken out of the data. The final data contained 757 valid respondents, averaging about 95 valid respondents per design version of the survey.

An MCMC method was used to obtain the estimates of $\theta^{*}$ in $(20)$ where a MetropolisHastings (M-H) algorithm was used within a Gibbs sampler to generate the posterior draws. Appendix $\mathrm{C}$ provides the details of the algorithm. Using the responses from part 1 and from each design version of part 2 of the study, the proposed hierarchical model was fit to the data and the posterior estimates of $\theta^{*}$ were obtained, as reported in Table 3 with the posterior means and the posterior standard deviations in parenthesis.

\begin{tabular}{||c|c|c|c|c||}
\hline$\theta^{*}$ & Design I & Design II & Design III & Design IV \\
\hline$\mu$ & 0.325 & 0.234 & 0.254 & 0.202 \\
& $(0.117)$ & $(0.115)$ & $(0.112)$ & $(0.112)$ \\
\hline$x_{\mathrm{A}}$ & 3.427 & 3.270 & 3.495 & 3.174 \\
& $(0.499)$ & $(0.512)$ & $(0.506)$ & $(0.520)$ \\
\hline$\nu_{\mathrm{A}}$ & 0.224 & 0.289 & 0.240 & 0.373 \\
& $(0.138)$ & $(0.150)$ & $(0.141)$ & $(0.143)$ \\
\hline$\omega_{\mathrm{A}}$ & 0.925 & 0.891 & 0.922 & 0.943 \\
& $(0.070)$ & $(0.092)$ & $(0.070)$ & $(0.054)$ \\
\hline$\theta_{\mathrm{P}}$ & -0.048 & 0.061 & -0.015 & -0.126 \\
& $(0.123)$ & $(0.125)$ & $(0.129)$ & $(0.120)$ \\
\hline$\theta_{\mathrm{C}}$ & 1.595 & 1.705 & 1.414 & 1.684 \\
& $(0.180)$ & $(0.175)$ & $(0.164)$ & $(0.172)$ \\
\hline$\theta_{\mathrm{T}}$ & 0.529 & 0.508 & 0.681 & 0.631 \\
& $(0.163)$ & $(0.163)$ & $(0.151)$ & $(0.153)$ \\
\hline
\end{tabular}

Table 3: Posterior Estimates of $\theta^{*}$ From Part 1 and Each Design Version of Part 2 of the Study

In all four design versions, the Bayesian 95\% highest probability density (HPD) regions of $\nu_{A}$ (the level effect of APR) do not include 0, confirming the existence of the level effect. That is, the contrast between the $8.99 \%$ and the $17.99 \%$ APR increases with the addition of the two intermediate APR levels. Estimation results on the other parameters are quite consistent in all occasions. For example, estimates on credit card provider $\left(\theta_{\mathrm{P}}\right)$ suggest that respondents do not have 
a preference of one provider (Capital One) over another (Citibank); estimates on rewards $\left(\theta_{\mathrm{C}}\right.$ and $\left.\theta_{\mathrm{T}}\right)$ suggest that respondents prefer cash reward over none and travel over none.

The parameter estimates reported in Table 3 indicate that the level effect parameter $\left(v_{\mathrm{A}}\right)$ is estimated to have a posterior mean of 0.28 on average, and that the range-frequency weight $\left(\omega_{\mathrm{A}}\right)$ in equation (16) has a posterior mean of 0.92 on average. This implies that attribute-levels in this study map onto the range-frequency value $J_{g}$ mostly through the relative range $R_{g}$. In addition, for each addition of an intermediate APR level, the mean perceived distance between the highest and lowest APR levels increases by 0.28. That is, the mean contrast between the highest and lowest APR in part 2 of the study is $0.28 * 2=0.56$ units larger than in part 1 of the study due to the addition of two intermediate APR levels. Thus, the level effect is estimated to be large relative to the other effect sizes -- approximately as large as the mean contrast between a travel reward versus no reward.

Accuracy of Estimates

We compare the efficacy of the four different designs using two measures of accuracy -- the determinant of the variance-covariance matrix (DETVAR) of the posterior estimates of $\theta^{*}$ normalized by the size of $\theta^{*}$, and the average variance (AVGVAR) of the posterior estimates of $\theta^{*}$ normalized by the size of $\theta^{*}$. Results are displayed in Table 4. We note that without knowledge of the actual values of $\theta^{*}$, these two measures provide direct measures of the estimation efficiency by assuming that the parameter estimates are unbiased.

Both the DETVAR and the AVGVAR measures show consistency with the relative $\widetilde{\psi}$ efficiencies of the designs, that is, designs with higher $\tilde{\psi}$-efficiency lead to smaller variances of the $\theta^{*}$ estimates as measured by DETVAR and AVGVAR. Table 4 also shows that neither of the performance measures is consistent with the relative D-efficiencies of the designs. Note especially designs II and IV, where design IV is more $\tilde{\psi}$-efficient but less D-efficient than design II. The 
performance measures suggest that the efficiency of hyperparameter estimation is more consistent with the $\widetilde{\psi}$-efficiency than the classical D-efficiency of a design, providing positive evidence for the use of the $\widetilde{\psi}$-criterion in designing experiments for efficient estimation of hyperparameters.

\begin{tabular}{||l|l|l|l|l||}
\hline \hline Performance Measure & $\begin{array}{l}\text { Design I } \\
\text { Rel. } \widetilde{\psi}=100 \% \\
\text { Rel. D=100\% }\end{array}$ & $\begin{array}{l}\text { Design II } \\
\text { Rel. } \widetilde{\psi}=95.5 \% \\
\text { Rel. D=100\% }\end{array}$ & $\begin{array}{l}\text { Design III } \\
\text { Rel. } \widetilde{\psi}=98.4 \% \\
\text { Rel. D=100\% }\end{array}$ & $\begin{array}{l}\text { Design IV } \\
\text { Rel. } \widetilde{\psi}=100.4 \% \\
\text { Rel. D =93.8\% }\end{array}$ \\
\hline $\begin{array}{l}\text { DETVAR } \\
\text { Relative DETVAR Efficiency }\end{array}$ & 0.013 & 0.017 & 0.014 & 0.013 \\
& $100 \%$ & $76.5 \%$ & $92.9 \%$ & $100 \%$ \\
\hline $\begin{array}{l}\text { AVGVAR } \\
\text { Relative AVGVAR Efficiency }\end{array}$ & 0.046 & 0.054 & $\begin{array}{l}0.051 \\
90.2 \%\end{array}$ & 0.046 \\
& $100 \%$ & $85.2 \%$ & $100 \%$ \\
\hline
\end{tabular}

Table 4: Performance Comparison of the Four Designs in Part 2 of the Study

\section{Predictive Performance}

Data from part 3 of the study were used as validation data to check how well the model predicts to a new context with a different number of APR levels. Estimates of $\theta^{*}$ obtained in Table 3 from each design $\mathrm{k}(\mathrm{k}=1, \ldots, 4)$ of part 2 of the study were used to predict the mean ratings of the 12 profiles in each design version $j(j=1,2,3)$ of part 3 of the study:

$$
\hat{\bar{y}}_{3 j k}=\int \mathrm{X}_{3 j} \mathrm{Z}_{3} \theta \times \pi\left(\theta, \Lambda_{1}, \Lambda_{2}, \sigma_{1}^{2}, \sigma_{2}^{2} \mid\left\{y_{1 i}, y_{2 i k}\right\}, \mathrm{X}_{1}, \mathrm{X}_{2 k}, \mathrm{Z}_{1}, \mathrm{Z}_{2}\right) d \theta d \Lambda_{1} d \Lambda_{2} d \sigma_{1}^{2} d \sigma_{2}^{2}
$$

where $\mathrm{X}_{3 \mathrm{j}}$ is the model matrix in design version $\mathrm{j}(\mathrm{j}=1,2,3)$ of part 3 of the study, as shown in Table B.5 of Appendix B. $\mathrm{X}_{1}$ is the model matrix in part 1 , and $\mathrm{X}_{2 \mathrm{k}}$ is the model matrix in design $\mathrm{k}$ $(\mathrm{k}=1, \ldots, 4)$ of part 2 of the study, as shown respectively in Table B.1 and B.3 of Appendix B. $y_{1 i}$ is the vector of responses from respondent $\mathrm{i}$ in part 1 , and $y_{2 i k}$ is the vector of responses from respondent $\mathrm{i}$ in design $\mathrm{k}$ of part 2 of the study. Appendix $\mathrm{D}$ provides the prediction results on the three design versions of part 3 of the study. For most profiles, the actual mean ratings were within 
the $95 \%$ Bayesian highest posterior density region of the predictions. Prediction accuracy is measured with expected squared error loss (MSE):

$$
\begin{aligned}
M S E_{j k}=\int & \left(\bar{y}_{3 j}-\mathrm{X}_{3 j} \mathrm{Z}_{3} \theta\right)^{\prime}\left(\bar{y}_{3 j}-\mathrm{X}_{3 j} \mathrm{Z}_{3} \theta\right) \\
& \times \pi\left(\theta, \Lambda_{1}, \Lambda_{2}, \sigma_{1}^{2}, \sigma_{2}^{2} \mid\left\{y_{1 i}, y_{2 i k}\right\}, \mathrm{X}_{1}, \mathrm{X}_{2 k}, \mathrm{Z}_{1}, \mathrm{Z}_{2}\right) d \theta d \Lambda_{1} d \Lambda_{2} d \sigma_{1}^{2} d \sigma_{2}^{2}
\end{aligned}
$$

where $\bar{y}_{3 j}$ is the actual mean ratings of the 12 profiles in design version $j(j=1,2,3)$ of part 3 of the study.

Table 5 summarizes the prediction accuracy on the validation data based on each of the four sets of $\theta^{*}$ estimates in Table 3. The MSE of the predictions based on the $\theta^{*}$ estimates from Design III is the smallest compared to the other designs selected for part 2 of the study, while the MSE of the predictions from Design II is the largest. The prediction MSE measure shows that designs with higher $\widetilde{\psi}$-efficiency lead to more accurate predictions in general, but not always. Since the $\widetilde{\psi}$ criterion is developed for parameter estimation and not for future prediction, it is reasonable to see that the DETVAR and the AVGVAR measures line up exactly in the same order as the relative $\widetilde{\psi}$ efficiency, but the prediction MSE measure does not. If the primary interest is on future predictions rather than hyperparameter estimation, then it is not efficient to use the $\tilde{\psi}$ criterion in designing the conjoint experiments. In such a situation, a criterion that focuses on future prediction needs to be developed for the model under consideration, for example, the V-and G- criterion discussed by Kessels et al. (2006) for non-hierarchical models.

\begin{tabular}{||l|l|l|l|l||}
\hline $\begin{array}{l}\text { Predictions to } \\
\text { Holdout }\end{array}$ & Design I & Design II & Design III & Design IV \\
\hline Version 1 & 0.189 & 0.210 & 0.138 & 0.199 \\
\hline Version 2 & 0.177 & 0.166 & 0.155 & 0.170 \\
\hline Version 3 & 0.226 & 0.251 & 0.191 & 0.199 \\
\hline All & 0.198 & 0.209 & 0.161 & 0.189 \\
\hline
\end{tabular}

Table 5: MSE of Mean Rating Predictions in Holdout Study 


\section{Discussion and Concluding Remarks}

The level effect is an example of a broad class of problems in Marketing and other disciplines that involve the learning of effect sizes and what drives big or small effects. Learning of the level effect gives us ideas on how consumer sensitivity to a product attribute varies with the number of attribute levels, and allows us to predict to a new context. We propose in this paper a hierarchical linear model that models individual consumer behavior and, by incorporating a number of ideas from the Psychology literature, models the level effect as a hyperparameter.

We designed a credit card survey study in which the survey designs were selected according to the $\widetilde{\psi}$-criterion introduced in (8) for efficient estimation of hyperparameters. The discussion and choice of design criteria for estimation of hyperparameters is an under-developed topic in marketing research and, with the advent of modern Bayesian statistical methods, the estimation of models characterizing the variation of effect sizes across contexts and environments is likely to become more prevalent. Our design criterion accommodates interactions between individual-level variables and contextual variables as reflected through matrices $X_{i}$ and $Z_{i}$ in hierarchical models. The interactions are present when studying contextual factors that influence consumer preference and choice. Our design criterion also nests the commonly used D-criterion for fixed-effects linear models as a special case.

From our survey data, we estimate a large contextual level effect that points to increased sensitivity to attribute-levels as the number of levels increases. The level-effect has implications for merchandising because it implies that consumer preference for product features is dependent on the assortment of choice. Our results imply that a high-assortment retailer will find that consumers express greater preference among attribute levels, while a low-assortment retailer will find that preferences are not as well defined and that consumers are more willing to switch among choice 
alternatives. This finding is consistent with the merchandising policies of discount retailers, such as WalMart, who typically offer low levels of assortment. We note that the level effect we examined is on a monotonic attribute, not one for which an ideal-point preference exists. We have not examined if the level effect exists for nominal attributes such as the color - e.g., that increasing choices of colors on cars makes people more sensitive to car color when making a purchase decision.

Hierarchical models offer a convenient structure for pooling results across studies to quantify changes in effect-sizes. These changes have typically not been associated with covariates, $\mathrm{Z}_{\mathrm{i}}$, that describe aspects of the study from which the data originate. For example, while researchers routinely compare responses for alternative stimuli, the comparison typically involves examination of simple contrasts, or, equivalently, assumes that $\mathrm{Z}_{\mathrm{i}}$ in the hierarchical model is dummy-variable coded. Hierarchical models allow for a more general coding scheme that enables the study of covariates that are more generally associated with experiment conditions, as illustrated in our empirical study. The design criterion presented here provides a means of evaluating designs for efficient learning about these contextual effects.

An interesting direction for future research is to investigate optimal designs under alternative design criteria that may be of interest, such as a criterion that focuses on future predictions, or a criterion that aims for efficient model discrimination. Additional topics for future research include the study of incomplete block designs in which different respondents receive different sets of stimuli. Furthermore, a natural extension of the current research is to the choice designs in the setting of hierarchical non-linear models. 


\section{APPENDIX A: The $\widetilde{\psi}$-criterion for Efficient Estimation of $\mathbf{g}(\boldsymbol{\theta})$ of hyperparameter vector $\boldsymbol{\theta}$}

We derive the $\widetilde{\psi}$-criterion following the same framework used by Liu et al. (2007). When the primary interest is in the estimation of a function $g(\theta)$ of hyperparameter vector $\theta$, we seek an optimal design with $\left\{\mathrm{X}_{\mathrm{i}}\right\}$ and $\left\{\mathrm{Z}_{\mathrm{i}}\right\}, \mathrm{i}=1, \ldots, \mathrm{n}$, that maximizes the expected gain in Shannon Information, that is, we seek a design that gives maximum

$$
\int\left\{\log p\left(g(\theta) \mid y, \mathrm{X}_{1}, \ldots, \mathrm{X}_{n}, Z_{1}, \ldots, Z_{n}\right)\right\} p\left(g(\theta) \mid y, \mathrm{X}_{1}, \ldots, \mathrm{X}_{n}, Z_{1}, \ldots, Z_{n}\right) p\left(y \mid \mathrm{X}_{1}, \ldots, \mathrm{X}_{n}, Z_{1}, \ldots, Z_{n}\right) d g(\theta) d y
$$

where $y=\left(y_{1}^{\prime}, \ldots, y_{n}^{\prime}\right)^{\prime}$. Using Berger (1985, pg 224, (iv)), and following Liu et al. (2007), the posterior distribution of $\theta \mid y, \mathrm{X}_{1}, \ldots, \mathrm{X}_{n}, Z_{1}, \ldots, Z_{n}$ is approximately normal with mean $\hat{\theta}$ and variancecovariance matrix

$$
\left[\sum_{i=1}^{n} \mathrm{Z}_{i}^{\prime} \mathrm{X}_{i}^{\prime}\left(\hat{\sigma}^{2} \mathrm{I}_{m_{i}}+\mathrm{X}_{i} \hat{\Lambda} \mathrm{X}_{i}^{\prime}\right)^{-1} \mathrm{X}_{i} \mathrm{Z}_{i}\right]^{-1}
$$

where $\hat{\theta}, \hat{\Lambda}$, and $\hat{\sigma}^{2}$ are the maximum likelihood estimates of $\theta, \Lambda$, and $\sigma^{2}$. Using first-order Taylor-series expansion, the distribution of $g(\theta) \mid y, \mathrm{X}_{1}, \ldots, \mathrm{X}_{n}, Z_{1}, \ldots, Z_{n}$ can be approximated by a normal distribution with mean $g(\hat{\theta})$, and variance-covariance matrix

$$
\left(\frac{\partial g(\theta)}{\partial \theta}\right)_{\theta=\hat{\theta}}^{\prime}\left[\sum_{i=1}^{n} \mathrm{Z}_{i}^{\prime} \mathrm{X}_{i}^{\prime}\left(\hat{\sigma}^{2} \mathrm{I}_{m_{i}}+\mathrm{X}_{i} \hat{\Lambda} \mathrm{X}_{i}^{\prime}\right)^{-1} \mathrm{X}_{i} \mathrm{Z}_{i}\right]^{-1}\left(\frac{\partial g(\theta)}{\partial \theta}\right)_{\theta=\hat{\theta}}
$$

Therefore, the maximization of (A.1) is approximately equivalent to the maximization of

$$
\int\left\{-\log \left|\left(\frac{\partial g(\theta)}{\partial \theta}\right)_{\theta=\hat{\theta}}^{\prime}\left(\sum_{i=1}^{n} \mathrm{Z}_{i}^{\prime} \mathrm{X}_{i}^{\prime}\left(\hat{\sigma}^{2} \mathrm{I}_{m_{i}}+\mathrm{X}_{i} \hat{\Lambda} \mathrm{X}_{i}^{\prime}\right)^{-1} \mathrm{X}_{i} \mathrm{Z}_{i}\right)^{-1}\left(\frac{\partial g(\theta)}{\partial \theta}\right)_{\theta=\hat{\theta}}\right|\right\} p\left(y \mid \mathrm{X}_{1}, \ldots, \mathrm{X}_{n}, Z_{1}, \ldots, Z_{n}\right) d y
$$

The integrand depends on y only through the consistent maximum likelihood estimates of $\theta, \Lambda$, and $\sigma^{2}$. Following Chaloner and Verdinelli (1995, pg. 286), a further approximation can be 
taken where the prior distribution of $\theta, \Lambda$, and $\sigma^{2}$ is used to approximate the distribution of $\hat{\theta}, \hat{\Lambda}$, and $\hat{\sigma}^{2}$, that is, (A.4) is further approximated by $\tilde{\psi}=\int\left\{-\log \left|\left(\frac{\partial g(\theta)}{\partial \theta}\right)\left(\sum_{i=1}^{n} \mathbf{Z}_{i}^{\prime} \mathbf{X}_{i}^{\prime}\left(\sigma^{2} \mathrm{I}_{m_{i}}+\mathrm{X}_{i} \Lambda \mathrm{X}_{i}^{\prime}\right)^{-1} \mathrm{X}_{i} \mathrm{Z}_{i}\right)^{-1}\left(\frac{\partial g(\theta)}{\partial \theta}\right)\right|\right\} p(\theta) p(\Lambda) p\left(\sigma^{2}\right) d \theta d \Lambda d \sigma^{2}$.

Therefore, for efficient estimation of a function $g(\theta)$ of hyperparameter vector $\theta$, we seek an optimal with $\left\{\mathrm{X}_{\mathrm{i}}\right\}$ and $\left\{\mathrm{Z}_{\mathrm{i}}\right\}, \mathrm{i}=1, \ldots, \mathrm{n}$, that maximizes (A.5), and we call the criterion $\widetilde{\psi}$-criterion.. 
APPENDIX B: Survey Designs and Corresponding Model Matrices

\begin{tabular}{lll} 
APR & Provider & Rewards \\
\hline $17.99 \%$ & Capital One & Travel \\
$17.99 \%$ & Capital One & Cash \\
$17.99 \%$ & Capital One & None \\
$17.99 \%$ & Citibank & Travel \\
$17.99 \%$ & Citibank & Cash \\
$17.99 \%$ & Citibank & None \\
$8.99 \%$ & Capital One & Travel \\
$8.99 \%$ & Capital One & Cash \\
$8.99 \%$ & Capital One & None \\
$8.99 \%$ & Citibank & Travel \\
$8.99 \%$ & Citibank & Cash \\
$8.99 \%$ & Citibank & None \\
\hline
\end{tabular}

\begin{tabular}{|c|c|c|c|c|}
\hline \multicolumn{5}{|c|}{ Corresponding Model Matrix $\mathrm{X}_{1}$} \\
\hline 1 & 0 & 1 & 0 & 1 \\
\hline 1 & 0 & 1 & 1 & 0 \\
\hline 1 & 0 & 1 & 0 & 0 \\
\hline 1 & 0 & 0 & 0 & 1 \\
\hline 1 & 0 & 0 & 1 & 0 \\
\hline 1 & 0 & 0 & 0 & 0 \\
\hline 1 & 1 & 1 & 0 & 1 \\
\hline 1 & 1 & 1 & 1 & 0 \\
\hline 1 & 1 & 1 & 0 & 0 \\
\hline 1 & 1 & 0 & 0 & 1 \\
\hline 1 & 1 & 0 & 1 & 0 \\
\hline 1 & 1 & 0 & 0 & 0 \\
\hline
\end{tabular}

Table B.1: Full-factorial design for stimuli construction in part 1 of the study and corresponding model matrix under dummy coding

DESIGN I

\begin{tabular}{lll} 
APR & Provider & Rewards \\
\hline $17.99 \%$ & Capital One & Cash \\
$17.99 \%$ & Citibank & Travel \\
$17.99 \%$ & Citibank & None \\
$15.99 \%$ & Capital One & Travel \\
$15.99 \%$ & Capital One & None \\
$15.99 \%$ & Citibank & Cash \\
$9.99 \%$ & Capital One & Travel \\
$9.99 \%$ & Capital One & Cash \\
$9.99 \%$ & Citibank & None \\
$8.99 \%$ & Capital One & None \\
$8.99 \%$ & Citibank & Travel \\
$8.99 \%$ & Citibank & Cash \\
\hline
\end{tabular}

\section{DESIGN III}

\begin{tabular}{lll} 
APR & Provider & Rewards \\
\hline $17.99 \%$ & Capital One & Travel \\
$17.99 \%$ & Citibank & Cash \\
$17.99 \%$ & Citibank & None \\
$15.99 \%$ & Capital One & Cash \\
$15.99 \%$ & Capital One & None \\
$15.99 \%$ & Citibank & Travel \\
$9.99 \%$ & Capital One & Cash \\
$9.99 \%$ & Citibank & Travel \\
$9.99 \%$ & Citibank & None \\
$8.99 \%$ & Capital One & Travel \\
$8.99 \%$ & Capital One & None \\
$8.99 \%$ & Citibank & Cash \\
\hline
\end{tabular}

DESIGN II

\begin{tabular}{lll} 
APR & Provider & Rewards \\
\hline $17.99 \%$ & Capital One & Travel \\
$17.99 \%$ & Capital One & Cash \\
$17.99 \%$ & Citibank & None \\
$15.99 \%$ & Capital One & None \\
$15.99 \%$ & Citibank & Travel \\
$15.99 \%$ & Citibank & Cash \\
$9.99 \%$ & Capital One & Travel \\
$9.99 \%$ & Capital One & Cash \\
$9.99 \%$ & Citibank & None \\
$8.99 \%$ & Capital One & None \\
$8.99 \%$ & Citibank & Travel \\
$8.99 \%$ & Citibank & Cash \\
\hline
\end{tabular}

\section{DESIGN IV}

\begin{tabular}{lll} 
APR & Provider & Rewards \\
\hline $17.99 \%$ & Capital One & Cash \\
$17.99 \%$ & Citibank & Travel \\
$17.99 \%$ & Citibank & None \\
$15.99 \%$ & Capital One & Travel \\
$15.99 \%$ & Capital One & None \\
$15.99 \%$ & Citibank & Cash \\
$9.99 \%$ & Capital One & Travel \\
$9.99 \%$ & Capital One & Cash \\
$9.99 \%$ & Citibank & None \\
$8.99 \%$ & Capital One & Cash \\
$8.99 \%$ & Capital One & None \\
$8.99 \%$ & Citibank & Travel \\
\hline
\end{tabular}

Table B.2: Four versions of designs for stimuli construction in part 2 of the study 


\section{Design I}

\begin{tabular}{lllllll}
\hline 1 & 0 & 0 & 0 & 1 & 1 & 0 \\
1 & 0 & 0 & 0 & 0 & 0 & 1 \\
1 & 0 & 0 & 0 & 0 & 0 & 0 \\
1 & 1 & 0 & 0 & 1 & 0 & 1 \\
1 & 1 & 0 & 0 & 1 & 0 & 0 \\
1 & 1 & 0 & 0 & 0 & 1 & 0 \\
1 & 0 & 1 & 0 & 1 & 0 & 1 \\
1 & 0 & 1 & 0 & 1 & 1 & 0 \\
1 & 0 & 1 & 0 & 0 & 0 & 0 \\
1 & 0 & 0 & 1 & 1 & 0 & 0 \\
1 & 0 & 0 & 1 & 0 & 0 & 1 \\
1 & 0 & 0 & 1 & 0 & 1 & 0 \\
\hline
\end{tabular}

\section{Design III}

\begin{tabular}{lllllll}
\hline 1 & 0 & 0 & 0 & 1 & 0 & 1 \\
1 & 0 & 0 & 0 & 0 & 1 & 0 \\
1 & 0 & 0 & 0 & 0 & 0 & 0 \\
1 & 1 & 0 & 0 & 1 & 1 & 0 \\
1 & 1 & 0 & 0 & 1 & 0 & 0 \\
1 & 1 & 0 & 0 & 0 & 0 & 1 \\
1 & 0 & 1 & 0 & 1 & 1 & 0 \\
1 & 0 & 1 & 0 & 0 & 0 & 1 \\
1 & 0 & 1 & 0 & 0 & 0 & 0 \\
1 & 0 & 0 & 1 & 1 & 0 & 1 \\
1 & 0 & 0 & 1 & 1 & 0 & 0 \\
1 & 0 & 0 & 1 & 0 & 1 & 0 \\
\hline
\end{tabular}

\section{Design II}

\begin{tabular}{lllllll}
\hline 1 & 0 & 0 & 0 & 1 & 0 & 1 \\
1 & 0 & 0 & 0 & 1 & 1 & 0 \\
1 & 0 & 0 & 0 & 0 & 0 & 0 \\
1 & 1 & 0 & 0 & 1 & 0 & 0 \\
1 & 1 & 0 & 0 & 0 & 0 & 1 \\
1 & 1 & 0 & 0 & 0 & 1 & 0 \\
1 & 0 & 1 & 0 & 1 & 0 & 1 \\
1 & 0 & 1 & 0 & 1 & 1 & 0 \\
1 & 0 & 1 & 0 & 0 & 0 & 0 \\
1 & 0 & 0 & 1 & 1 & 0 & 0 \\
1 & 0 & 0 & 1 & 0 & 0 & 1 \\
1 & 0 & 0 & 1 & 0 & 1 & 0 \\
\hline
\end{tabular}

\begin{tabular}{ccccccc}
\multicolumn{8}{c}{ Design IV } \\
\hline 1 & 0 & 0 & 0 & 1 & 1 & 0 \\
1 & 0 & 0 & 0 & 0 & 0 & 1 \\
1 & 0 & 0 & 0 & 0 & 0 & 0 \\
1 & 1 & 0 & 0 & 1 & 0 & 1 \\
1 & 1 & 0 & 0 & 1 & 0 & 0 \\
1 & 1 & 0 & 0 & 0 & 1 & 0 \\
1 & 0 & 1 & 0 & 1 & 0 & 1 \\
1 & 0 & 1 & 0 & 1 & 1 & 0 \\
1 & 0 & 1 & 0 & 0 & 0 & 0 \\
1 & 0 & 0 & 1 & 1 & 1 & 0 \\
1 & 0 & 0 & 1 & 1 & 0 & 0 \\
1 & 0 & 0 & 1 & 0 & 0 & 1 \\
\hline
\end{tabular}

Table B.3: Corresponding model matrix $\left(\mathrm{X}_{2}\right.$, dummy-coded) in part 2 of the study

\begin{tabular}{lll}
\multicolumn{3}{c}{ DESIGN I } \\
APR & Provider & Rewards \\
\hline $17.99 \%$ & CapOne & Cash \\
$17.99 \%$ & CapOne & None \\
$17.99 \%$ & Citibank & Travel \\
$17.99 \%$ & Citibank & Cash \\
$9.99 \%$ & CapOne & Travel \\
$9.99 \%$ & CapOne & None \\
$9.99 \%$ & Citibank & Cash \\
$9.99 \%$ & Citibank & None \\
$8.99 \%$ & CapOne & Travel \\
$8.99 \%$ & CapOne & Cash \\
$8.99 \%$ & Citibank & Travel \\
$8.99 \%$ & Citibank & None \\
\hline
\end{tabular}

\begin{tabular}{|lll}
\multicolumn{3}{c}{ DESIGN II } \\
APR & Provider & Rewards \\
\hline $17.99 \%$ & CapOne & Travel \\
$17.99 \%$ & CapOne & None \\
$17.99 \%$ & Citibank & Cash \\
$17.99 \%$ & Citibank & None \\
$9.99 \%$ & CapOne & Cash \\
$9.99 \%$ & CapOne & None \\
$9.99 \%$ & Citibank & Travel \\
$9.99 \%$ & Citibank & Cash \\
$8.99 \%$ & CapOne & Travel \\
$8.99 \%$ & CapOne & Cash \\
$8.99 \%$ & Citibank & Travel \\
$8.99 \%$ & Citibank & None \\
\hline & &
\end{tabular}

\begin{tabular}{lll}
\multicolumn{3}{|c}{ DESIGN III } \\
APR & Provider & Rewards \\
\hline $17.99 \%$ & CapOne & Cash \\
$17.99 \%$ & CapOne & None \\
$17.99 \%$ & Citibank & Travel \\
$17.99 \%$ & Citibank & Cash \\
$17.99 \%$ & Citibank & None \\
$9.99 \%$ & CapOne & Travel \\
$9.99 \%$ & CapOne & None \\
$9.99 \%$ & Citibank & Cash \\
$8.99 \%$ & CapOne & Travel \\
$8.99 \%$ & CapOne & Cash \\
$8.99 \%$ & Citibank & Travel \\
$8.99 \%$ & Citibank & None \\
\hline
\end{tabular}

Table B.4: Three versions of designs for stimuli construction in part 3 of the study 


\begin{tabular}{cccccc}
\multicolumn{7}{c}{ Design I } \\
\hline 1 & 0 & 0 & 1 & 1 & 0 \\
1 & 0 & 0 & 1 & 0 & 0 \\
1 & 0 & 0 & 0 & 0 & 1 \\
1 & 0 & 0 & 0 & 1 & 0 \\
1 & 1 & 0 & 1 & 0 & 1 \\
1 & 1 & 0 & 1 & 0 & 0 \\
1 & 1 & 0 & 0 & 1 & 0 \\
1 & 1 & 0 & 0 & 0 & 0 \\
1 & 0 & 1 & 1 & 0 & 1 \\
1 & 0 & 1 & 1 & 1 & 0 \\
1 & 0 & 1 & 0 & 0 & 1 \\
1 & 0 & 1 & 0 & 0 & 0 \\
\hline
\end{tabular}

\begin{tabular}{cccccc}
\multicolumn{7}{c}{ Design II } \\
\hline 1 & 0 & 0 & 1 & 0 & 1 \\
1 & 0 & 0 & 1 & 0 & 0 \\
1 & 0 & 0 & 0 & 1 & 0 \\
1 & 0 & 0 & 0 & 0 & 0 \\
1 & 1 & 0 & 1 & 1 & 0 \\
1 & 1 & 0 & 1 & 0 & 0 \\
1 & 1 & 0 & 0 & 0 & 1 \\
1 & 1 & 0 & 0 & 1 & 0 \\
1 & 0 & 1 & 1 & 0 & 1 \\
1 & 0 & 1 & 1 & 1 & 0 \\
1 & 0 & 1 & 0 & 0 & 1 \\
1 & 0 & 1 & 0 & 0 & 0 \\
\hline
\end{tabular}

\begin{tabular}{cccccc}
\multicolumn{8}{c}{ Design III } \\
\cline { 2 - 6 } & 0 & 0 & 1 & 1 & 0 \\
1 & 0 & 0 & 1 & 0 & 0 \\
1 & 0 & 0 & 0 & 0 & 1 \\
1 & 0 & 0 & 0 & 1 & 0 \\
1 & 0 & 0 & 0 & 0 & 0 \\
1 & 1 & 0 & 1 & 0 & 1 \\
1 & 1 & 0 & 1 & 0 & 0 \\
1 & 1 & 0 & 0 & 1 & 0 \\
1 & 0 & 1 & 1 & 0 & 1 \\
1 & 0 & 1 & 1 & 1 & 0 \\
1 & 0 & 1 & 0 & 0 & 1 \\
1 & 0 & 1 & 0 & 0 & 0 \\
\hline
\end{tabular}

Table B.5: Corresponding model matrix $\left(X_{3}\right.$, dummy-coded) in part 3 of the study

Study 1 Covariate Matrix $\mathrm{Z}_{1}$

\begin{tabular}{llllllll}
\hline 1 & 0 & 0 & 0 & 0 & 0 & 0 & 0 \\
0 & 1 & 2 & 0 & 0 & 0 & 0 & 0 \\
0 & 0 & 0 & 0 & 0 & 1 & 0 & 0 \\
0 & 0 & 0 & 0 & 0 & 0 & 1 & 0 \\
0 & 0 & 0 & 0 & 0 & 0 & 0 & 1 \\
\hline
\end{tabular}

Study 3 Covariate Matrix $\mathbf{Z}_{\mathbf{3}}$

\begin{tabular}{rrrrrrrr}
\hline 1 & 0 & 0 & 0 & 0 & 0 & 0 & 0 \\
0 & 0.5 & 1.5 & 0.39 & 1.17 & 0 & 0 & 0 \\
0 & 1 & 3 & 0 & 0 & 0 & 0 & 0 \\
0 & 0 & 0 & 0 & 0 & 1 & 0 & 0 \\
0 & 0 & 0 & 0 & 0 & 0 & 1 & 0 \\
0 & 0 & 0 & 0 & 0 & 0 & 0 & 1 \\
\hline
\end{tabular}

Study 2 Covariate Matrix $\mathrm{Z}_{2}$

\begin{tabular}{rrrrrrrr}
\hline 1 & 0 & 0 & 0 & 0 & 0 & 0 & 0 \\
0 & 0.33 & 1.33 & -0.11 & -0.44 & 0 & 0 & 0 \\
0 & 0.67 & 2.67 & 0.22 & 0.88 & 0 & 0 & 0 \\
0 & 1 & 4 & 0 & 0 & 0 & 0 & 0 \\
0 & 0 & 0 & 0 & 0 & 1 & 0 & 0 \\
0 & 0 & 0 & 0 & 0 & 0 & 1 & 0 \\
0 & 0 & 0 & 0 & 0 & 0 & 0 & 1 \\
\hline
\end{tabular}

Table B.6: Covariate matrices for the three parts of the study 


\section{APPENDIX C: MCMC Algorithm for Model Fitting}

The following standard prior distribution assumptions (see, for example, Gamerman, 1997; Rossi, Allenby and McCulloch, 2005) are made on parameters $\omega_{\mathrm{A}}, \theta_{-\omega_{A}}^{*}=\left(\mu, \kappa_{A}, v_{A}, \theta_{P}, \theta_{C}, \theta_{T}\right)^{\prime},\left\{\Lambda_{s}\right\}$ and $\left\{\sigma_{s}^{2}\right\}(s=1,2)$ :

$$
\begin{gathered}
\omega_{\mathrm{A}} \sim \operatorname{Uniform}(0,1) \\
\theta_{-\omega_{A}}^{*} \sim \operatorname{Normal}\left(b_{0}=0, D_{0}^{-1}=100 \mathrm{I}_{6}\right) \\
\Lambda_{s} \sim \text { Inverted Wishart }\left(\eta_{0, s}=p_{s}+3, \Delta_{0, s}=\eta_{0, s} \mathrm{I}_{p_{s}}\right) \\
\sigma_{s}^{2} \sim \text { Inverse Gamma }\left(\frac{w_{0}}{2}, \frac{W_{0, s}}{2}\right)
\end{gathered}
$$

Where $p_{1}=5, p_{2}=7, w_{0}=3$, and $W_{0, s}=w_{0}^{*} \sum_{i=1}^{n} \sum_{j=1}^{m_{i}}\left(y_{i j}-\bar{y}_{. .}\right)^{2} /\left(m_{s} n_{s}-1\right)$. Here, $m_{\mathrm{s}}$ represents the number of profiles and $n_{\mathrm{s}}$ represents the number of respondents in the study $\mathrm{s}(\mathrm{s}=1,2)$. In the 12 profile credit card survey studies 1 and $2, m_{1}=m_{2}=12$. A Metropolis-Hastings $(\mathrm{M}-\mathrm{H})$ algorithm is used within a Gibbs sampler. The estimation algorithm proceeds by recursively generating draws from the following densities:

1. Independently generate $\left\{\beta_{i, s}, i=1, \ldots, n, s=1,2\right\}$ from the following multivariate normal distribution:

$$
\begin{gathered}
{\left[\beta_{i, s} \mid y_{i, s}, X_{i, s}, Z_{s}, \theta, \Lambda_{s}, \sigma_{s}^{2}\right] \propto\left[y_{i, s} \mid X_{i, s}, \beta_{i, s}, \sigma_{s}^{2}\right]\left[\beta_{i, s} \mid Z_{s}, \theta, \Lambda_{s}\right] \sim \operatorname{Normal}\left(b_{i, s}, D_{i, s}\right),} \\
D_{i, s}=\left(\sigma_{s}^{-2} X_{i, s}^{\prime} X_{i, s}+\Lambda_{s}^{-1}\right)^{-1}, \quad b_{i, s}=D_{i, s}\left(\sigma_{s}^{-2} X_{i, s}^{\prime} y_{i, s}+\Lambda_{s}^{-1} Z_{s} \theta\right) .
\end{gathered}
$$

2. Generate $\left\{\sigma_{s}^{2}, s=1,2\right\}$ from the following Inverted chi-squared distribution 


$$
\begin{gathered}
{\left[\sigma_{s}^{2} \mid\left\{y_{i, s}, X_{i, s}, \beta_{i, s}\right\}\right] \propto\left[\left\{y_{i, s}\right\} \mid\left\{X_{i, s}, \beta_{i, s}, \sigma_{s}^{2}\right\}\right]\left[\sigma_{s}^{2}\right] \sim \text { Inverted Chi-squared }\left(w_{s}, W_{s}\right)} \\
w_{s}=w_{0, s}+m_{s} n_{s}, \quad W_{s}^{-1}=W_{0, s}^{-1}+\sum_{i=1}^{n_{s}}\left(y_{i, s}-X_{i, s} \beta_{i, s}\right)^{\prime}\left(y_{i, s}-X_{i, s} \beta_{i, s}\right) .
\end{gathered}
$$

\section{Generate $\theta_{-\omega_{A}}^{*}$}

$$
\left[\theta_{-\omega_{A}}^{*} \mid\left\{Z_{s}, \beta_{i, s}, \Lambda_{s}\right\}, \omega_{A}\right] \propto\left[\left\{\beta_{i, s}\right\} \mid\left\{Z_{s}, \Lambda_{s}\right\}, \theta\right]\left[\theta_{-\omega_{A}}^{*}\right]
$$

where $\left[\left\{\beta_{i, s}\right\} \mid\left\{Z_{s}, \Lambda_{s}\right\}, \theta\right]$ is the product of multivariate normal densities, $\prod_{s=1}^{S} \prod_{i=1}^{n}\left[\beta_{i, s} \mid Z_{s}, \theta, \Lambda_{s}\right]$.

A random-walk $\mathrm{M}-\mathrm{H}$ is used to generate the draws of $\theta_{-\omega_{A}}^{*}$. Let

$$
\theta_{-\omega_{A}}^{*(k)}=\left(\mu^{(k)}, \kappa_{A}^{(k)}, v_{A}^{(k)}, \theta_{P}^{(k)}, \theta_{C}^{(k)}, \theta_{T}^{(k)}\right)^{\prime}
$$

be the kth draw; the next draw is given by

$$
\theta_{-\omega_{A}}^{*(k+1)}=\theta_{-\omega_{A}}^{*(k)}+\delta
$$

where $\delta$ is a draw from the candidate generating density $\operatorname{Normal}\left(0,0.05^{2} \mathrm{I}\right)$. Let

$$
\theta^{(k)}=\left(\mu^{(k)}, \kappa_{A}^{(k)}, v_{A}^{(k)}, \kappa_{A}^{(k)} \omega_{A}, v_{A}^{(k)} \omega_{A}, \theta_{P}^{(k)}, \theta_{C}^{(k)}, \theta_{T}^{(k)}\right)^{\prime}
$$

The probability of accepting the new $\operatorname{draw} \theta_{-\omega_{A}}^{*(k+1)}$ is given by

$$
\min \left[\frac{\left[\left\{\beta_{i, s}\right\} \mid\left\{Z_{s}, \Lambda_{s}\right\}, \theta^{(k+1)}\right]\left[\theta_{-\omega_{A}}^{*(k+1)}\right]}{\left[\left\{\beta_{i, s}\right\} \mid\left\{Z_{s}, \Lambda_{s}\right\}, \theta^{(k)}\right]\left[\theta_{-\omega_{A}}^{*(k)}\right]}, \quad 1\right] .
$$




\section{Generate $\omega_{\mathrm{A}}$}

$$
\left[\omega_{A} \mid\left\{Z_{s}, \beta_{i, s}, \Lambda_{s}\right\}\right] \propto\left[\left\{\beta_{i, s}\right\} \mid\left\{Z_{s}, \Lambda_{s}\right\}, \theta\right]\left[\omega_{A}\right],
$$

where $\left[\left\{\beta_{i, s}\right\} \mid Z_{s}, \theta, \Lambda_{s}\right]$ is the product of multivariate normal densities, $\prod_{s=1}^{S} \prod_{i=1}^{n}\left[\beta_{i, s} \mid Z_{s}, \theta, \Lambda_{s}\right]$.

An Independent $\mathrm{M}-\mathrm{H}$ algorithm is used to generate the draws of $\omega_{\tau}$. Generate draws independently

from the Uniform $(0,1)$ distribution. Let $\omega_{A}^{(k)}$ be the kth draw, and $\omega_{A}^{(k+1)}$ be the next draw. Let

$$
\theta^{(k)}=\left(\mu, \kappa_{A}, v_{A}, \kappa_{A} \omega_{A}^{(k)}, v_{A} \omega_{A}^{(k)}, \theta_{P}, \theta_{C}, \theta_{T}\right)^{\prime}
$$

The probability of accepting the new draw $\omega_{\tau}^{(k+1)}$ is given by

$$
\min \left[\frac{\left[\left\{\beta_{i, s}\right\} \mid\left\{Z_{s}, \Lambda_{s}\right\}, \theta^{(k+1)}\right]}{\left[\left\{\beta_{i, s}\right\} \mid\left\{Z_{s}, \Lambda_{s}\right\}, \theta^{(k)}\right]}, \quad 1\right] .
$$

5. Generate $\left\{\Lambda_{s}, s=1, \ldots, S\right\}$ from the Inverted Wishart Distribution

$$
\begin{gathered}
{\left[\Lambda_{s} \mid\left\{\beta_{i, s}\right\}, Z_{s}, \theta\right] \propto\left[\left\{\beta_{i, s}\right\} \mid Z_{s}, \theta, \Lambda_{s}\right]\left[\Lambda_{s}\right] \sim \text { Inverted Wishart }\left(\eta_{s}, \Delta_{s}\right)} \\
\eta_{s}=\eta_{0, s}+n_{s}, \quad \Delta_{s}^{-1}=\Delta_{0, s}^{-1}+\sum_{i=1}^{n_{s}}\left(\beta_{i, s}-Z_{s} \theta\right)\left(\beta_{i, s}-Z_{s} \theta\right)^{\prime}
\end{gathered}
$$

300,000 iterations are run and every $30^{\text {th }}$ - iteration is kept. Posterior means and variances are calculated using draws after the burn-in period of the first 60,000 iterations. 
APPENDIX D: Prediction Validation Results

\begin{tabular}{|c|c|c|c|c|}
\hline $\begin{array}{c}\text { Actual } \\
\bar{y}_{3}\end{array}$ & $\begin{array}{l}\overline{\bar{y}}_{3} \text { from } \\
\text { Design I }\end{array}$ & $\begin{array}{l}\overline{\bar{y}}_{3} \text { from } \\
\text { Design II }\end{array}$ & $\begin{array}{l}\widehat{\bar{y}}_{3} \text { from } \\
\text { Design III }\end{array}$ & $\begin{array}{l}\overline{\bar{y}}_{3} \text { from } \\
\text { Design IV }\end{array}$ \\
\hline \multirow[t]{2}{*}{1.287} & 1.885 & 2.001 & 1.653 & 1.756 \\
\hline & $(0.224)$ & $(0.219)$ & (0.197) & $(0.196)$ \\
\hline \multirow[t]{2}{*}{0.681} & 0.281 & 0.295 & 0.238 & 0.071 \\
\hline & $(0.146)$ & $(0.135)$ & $(0.139)$ & $(0.138)$ \\
\hline \multirow[t]{2}{*}{1.011} & 0.863 & 0.743 & 0.935 & 0.828 \\
\hline & $(0.177)$ & $(0.171)$ & $(0.156)$ & $(0.163)$ \\
\hline \multirow[t]{2}{*}{1.383} & 1.934 & 1.940 & 1.668 & 1.885 \\
\hline & $(0.193)$ & $(0.190)$ & $(0.172)$ & $(0.181)$ \\
\hline \multirow[t]{2}{*}{4.043} & 4.326 & 4.307 & 4.541 & 4.437 \\
\hline & $(0.227)$ & $(0.240)$ & $(0.219)$ & $(0.209)$ \\
\hline \multirow[t]{2}{*}{3.362} & 3.793 & 3.799 & 3.860 & 3.810 \\
\hline & $(0.197)$ & $(0.208)$ & $(0.190)$ & $(0.182)$ \\
\hline \multirow[t]{2}{*}{5.000} & 5.447 & 5.443 & 5.290 & 5.624 \\
\hline & $(0.220)$ & $(0.228)$ & $(0.207)$ & $(0.197)$ \\
\hline \multirow[t]{2}{*}{3.351} & 3.843 & 3.738 & 3.876 & 3.939 \\
\hline & $(0.192)$ & $(0.212)$ & $(0.187)$ & $(0.174)$ \\
\hline \multirow[t]{2}{*}{5.181} & 4.898 & 4.940 & 5.134 & 5.004 \\
\hline & $(0.214)$ & $(0.235)$ & $(0.204)$ & $(0.209)$ \\
\hline \multirow[t]{2}{*}{5.745} & 5.969 & 6.137 & 5.868 & 6.062 \\
\hline & $(0.214)$ & $(0.216)$ & $(0.197)$ & $(0.196)$ \\
\hline \multirow[t]{2}{*}{5.330} & 4.948 & 4.879 & 5.150 & 5.133 \\
\hline & $(0.218)$ & $(0.236)$ & $(0.207)$ & $(0.206)$ \\
\hline \multirow[t]{2}{*}{4.436} & 4.415 & 4.371 & 4.469 & 4.506 \\
\hline & (0.187) & $(0.209)$ & (0.184) & (0.178) \\
\hline
\end{tabular}

Table D.1: Predictions of mean profile ratings in holdout study version 1 


\begin{tabular}{|c|c|c|c|c|}
\hline $\begin{array}{c}\text { Actual } \\
\bar{y}_{3}\end{array}$ & $\begin{array}{l}\overline{\bar{y}}_{3} \text { from } \\
\text { Design I }\end{array}$ & $\begin{array}{l}\overline{\bar{y}}_{3} \text { from } \\
\text { Design II }\end{array}$ & $\begin{array}{l}\widehat{\bar{y}}_{3} \text { from } \\
\text { Design III }\end{array}$ & $\begin{array}{l}\overline{\bar{y}}_{3} \text { from } \\
\text { Design IV }\end{array}$ \\
\hline \multirow[t]{2}{*}{0.979} & 0.814 & 0.804 & 0.919 & 0.698 \\
\hline & $(0.193)$ & $(0.190)$ & $(0.180)$ & $(0.180)$ \\
\hline \multirow[t]{2}{*}{0.511} & 0.281 & 0.295 & 0.238 & 0.071 \\
\hline & $(0.146)$ & $(0.135)$ & $(0.139)$ & $(0.138)$ \\
\hline \multirow[t]{2}{*}{1.043} & 1.934 & 1.940 & 1.668 & 1.885 \\
\hline & $(0.193)$ & $(0.190)$ & $(0.172)$ & $(0.181)$ \\
\hline \multirow[t]{2}{*}{0.543} & 0.330 & 0.234 & 0.254 & 0.201 \\
\hline & $(0.115)$ & $(0.115)$ & $(0.112)$ & $(0.113)$ \\
\hline \multirow[t]{2}{*}{5.532} & 5.397 & 5.504 & 5.275 & 5.495 \\
\hline & $(0.235)$ & $(0.240)$ & $(0.216)$ & $(0.203)$ \\
\hline \multirow[t]{2}{*}{3.755} & 3.793 & 3.799 & 3.860 & 3.810 \\
\hline & $(0.197)$ & $(0.208)$ & $(0.190)$ & $(0.182)$ \\
\hline \multirow[t]{2}{*}{4.426} & 4.376 & 4.246 & 4.557 & 4.566 \\
\hline & $(0.227)$ & $(0.240)$ & $(0.213)$ & $(0.204)$ \\
\hline \multirow[t]{2}{*}{5.383} & 5.447 & 5.443 & 5.290 & 5.624 \\
\hline & $(0.220)$ & $(0.228)$ & $(0.207)$ & $(0.197)$ \\
\hline \multirow[t]{2}{*}{5.277} & 4.898 & 4.940 & 5.134 & 5.004 \\
\hline & $(0.214)$ & $(0.235)$ & $(0.204)$ & $(0.209)$ \\
\hline \multirow[t]{2}{*}{6.468} & 5.969 & 6.137 & 5.868 & 6.062 \\
\hline & $(0.214)$ & $(0.216)$ & $(0.197)$ & $(0.196)$ \\
\hline \multirow[t]{2}{*}{5.277} & 4.948 & 4.879 & 5.150 & 5.133 \\
\hline & $(0.218)$ & $(0.236)$ & $(0.207)$ & $(0.206)$ \\
\hline \multirow[t]{2}{*}{4.415} & 4.415 & 4.371 & 4.469 & 4.506 \\
\hline & $(0.187)$ & $(0.209)$ & $(0.184)$ & $(0.178)$ \\
\hline
\end{tabular}

Table D.2: Predictions of mean profile ratings in holdout study version 2 


\begin{tabular}{|c|c|c|c|c|}
\hline $\begin{array}{c}\text { Actual } \\
\bar{y}_{3}\end{array}$ & $\begin{array}{l}\overline{\bar{y}}_{3} \text { from } \\
\text { Design I }\end{array}$ & $\begin{array}{l}\overline{\bar{y}}_{3} \text { from } \\
\text { Design II }\end{array}$ & $\begin{array}{l}\bar{y}_{3} \text { from } \\
\text { Design III }\end{array}$ & $\begin{array}{l}\overline{\bar{y}}_{3} \text { from } \\
\text { Design IV }\end{array}$ \\
\hline \multirow[t]{2}{*}{1.354} & 1.885 & 2.001 & 1.653 & 1.756 \\
\hline & $(0.224)$ & $(0.219)$ & $(0.197)$ & $(0.196)$ \\
\hline \multirow[t]{2}{*}{0.594} & 0.281 & 0.295 & 0.238 & 0.071 \\
\hline & $(0.146)$ & $(0.135)$ & $(0.139)$ & $(0.138)$ \\
\hline \multirow[t]{2}{*}{1.177} & 0.863 & 0.743 & 0.935 & 0.828 \\
\hline & $(0.177)$ & $(0.171)$ & $(0.156)$ & $(0.163)$ \\
\hline \multirow[t]{2}{*}{1.208} & 1.934 & 1.940 & 1.668 & 1.885 \\
\hline & (0.193) & $(0.190)$ & $(0.172)$ & $(0.181)$ \\
\hline \multirow[t]{2}{*}{0.583} & 0.330 & 0.234 & 0.254 & 0.201 \\
\hline & $(0.115)$ & $(0.115)$ & $(0.112)$ & $(0.113)$ \\
\hline \multirow[t]{2}{*}{4.250} & 4.326 & 4.307 & 4.541 & 4.437 \\
\hline & $(0.227)$ & $(0.240)$ & $(0.219)$ & $(0.209)$ \\
\hline \multirow[t]{2}{*}{3.490} & 3.793 & 3.799 & 3.860 & 3.810 \\
\hline & $(0.197)$ & $(0.208)$ & $(0.190)$ & $(0.182)$ \\
\hline \multirow[t]{2}{*}{5.646} & 5.447 & 5.443 & 5.290 & 5.624 \\
\hline & $(0.220)$ & $(0.228)$ & $(0.207)$ & $(0.197)$ \\
\hline \multirow[t]{2}{*}{5.135} & 4.898 & 4.940 & 5.134 & 5.004 \\
\hline & $(0.214)$ & $(0.235)$ & $(0.204)$ & $(0.209)$ \\
\hline \multirow[t]{2}{*}{6.583} & 5.969 & 6.137 & 5.868 & 6.062 \\
\hline & $(0.214)$ & $(0.216)$ & (0.197) & $(0.196)$ \\
\hline \multirow[t]{2}{*}{5.427} & 4.948 & 4.879 & 5.150 & 5.133 \\
\hline & $(0.218)$ & $(0.236)$ & $(0.207)$ & $(0.206)$ \\
\hline \multirow[t]{2}{*}{5.063} & 4.415 & 4.371 & 4.469 & 4.506 \\
\hline & $(0.187)$ & $(0.209)$ & $(0.184)$ & $(0.178)$ \\
\hline
\end{tabular}

Table D.3: Predictions of mean profile ratings in holdout study version 3 


\section{References}

Arora, Neeraj and Joel Huber (2001), "Improving Parameter Estimates and Model Prediction by Aggregate Customization in Choice Experiments," Journal of Consumer Research, 28 (September), $273-283$.

Atkinson, A.C. and A.N. Donev (1992), Optimum Experimental Designs, Clarendon Press, Oxford.

Berger, James O. (1985), Statistical Decision Theory and Bayesian Analysis, New York: Springer.

Chaloner, Kathryn and Isabella Verdinelli (1995), "Bayesian Experimental Design: A Review," Statistical Science, 10 (3), 273-304.

Cooke, Alan D.J., Chris Janiszewski, Marcus Cunha Jr., Suzanne A. Nasco, and Els De Wilde (2004), "Stimulus Context and the Formation of Consumer Ideals," Journal of Consumer Research, 31 (June), 112-124.

Creyer, Elizabeth and William T. Ross (1988), "The Effects of Range-Frequency manipulations on Conjoint Importance Weight Stability," Advances in Consumer Research, 15, 505-509.

Currim, Imran S., Charles B. Weinberg, and Dick.R. Wittink (1981), "Design of Subscription Programs for a Performing Arts Series," Journal of Consumer Research, 8 (June), 67-75.

Gamerman, Dani (1997), Markov Chain Monte Carlo, Chapman \& Hall.

Han, Cong and Kathryn Chaloner (2004), "Bayesian Experimental Design for Nonlinear Mixedeffects Models with Applications to HIV Dynamics," Biometrics, 60, 25-33.

Huber, Joel, John W. Payne, and Christopher Puto (1982), “Adding Asymmetrically Dominated Alternatives: Violations of Regularity and the Similarity Hypothesis," Journal of Consumer Research, 9 (June), 90-98.

Janiszewski, Chris and Donald R. Lichtenstein (1999), “A Range Theory Account of Price Perception,” Journal of Consumer Research, 25 (March), 353-368.

Kahn, Barbara, William L. Moore, and Rashi Glazer (1987), "Experiments in Constrained Choice,” Journal of Consumer Research, 14 (June), 96-113.

Kessels, Roselinde, Peter Goos, and Martina Vandebroek (2006), "A Comparison of Criteria to Design Efficient Choice Experiments," Journal of Marketing Research, 43 (3), 409-419.

Krumhansl, Carol L. (1978), "Concerning the Applicability of Geometric Models to Similarity Data: The Interrelationship Between Similarity and Spatial Density," Psychological Review, 85 (5), $445-463$.

Kuhfeld, Warren F (2005), "Experimental Design, Efficiency, Coding and Choice Designs," Tech. rep., SAS TS-722C, http://support.sas.com/techsup/tnote/tnote stat.html.

Kuhfeld, Warren F., Randall D. Tobias, and Mark Garratt (1994), "Efficient Experimental Design With Marketing Research Applications," Journal of Marketing Research, 31 (4), 545-557.

Lenk, Peter J., Wayne S. Desarbo, Paul E. Green, and Martin R. Young (1996), “Hierarchical Bayes Conjoint Analysis: Recovery of Partworth Heterogeneity from Reduced Experimental Designs," Marketing Science, 15 (2), 173-191. 
Liu, Qing, Angela M. Dean, and Greg M. Allenby (2007), “Optimal Experimental Designs for Hyperparameter Estimation in Hierarchical Linear Models," http://www.stat.osu.edu/ amd/dissertations.html, Submitted for publication.

Lynch, John G., Dipankar Chakravarti, and Anusree Mitra (1991), “Contrast Effects in Consumer Judgments: Changes in Mental Representations or in the Anchoring of Rating Scales?" Journal of Consumer Research, 18 (December), 284-297.

Mentre, France, Alain Mallet and Doha Baccar (1997), "Optimal Design in Random-Effects Regression Models," Biometrika, 84 (2), 429-442.

Parducci, Allen (1965), “Category Judgment: A range-frequency model,” Psychological Review, 72, 407-418.

(1974), "Contextual Effects: A Range-Frequency Analysis," in Handbook of Perception, eds. Edward Carterette and Morton Friedman, New York: Academic Press, 127-141.

(1982), "Category Ratings: Still more contextual effects," in Social Attitudes and Psychophysical Measurement, eds. Wegener B., Hillsdale, NJ: Erlbaum, 89-105.

Rossi, Peter E., Greg M. Allenby, and Robert McCulloch (2005), Bayesian Statistics and Marketing, John Wiley and Sons, Ltd.

Sandor, Zsolt and Michel Wedel (2001), "Designing Conjoint Choice Experiments Using Managers Prior Beliefs," Journal of Marketing Research, 38 (4), 430-444.

and (2002), "Profile Construction in Experimental Choice Designs for Mixed Logit Models," Marketing Science, 21 (4), 455-475. and -(2005), "Differentiated Bayesian Conjoint Choice Designs," Journal of Marketing Research, 55 (2), 210-218.

Simonson, Itamar and Amos Tversky (1992), "Choice in Context: Tradeoff Contrast and Extremeness Aversion," Journal of Marketing Research, 29 (3), 281-295.

Steenkamp, Jan-Benedict E.M. and Dick R. Wittink (1994), “The Metric Quality of Full-Profile Judgments and the Number-of-attribute-levels Effect in Conjoint Analysis," International Journal of Research in Marketing, 11, 275-286.

Tod, Michel, France Mentre, Yann Merle, and Alain Mallet (1998), "Robust Optimal Design for the Estimation of Hyperparameters in Population Pharmacokinetics," Journal of Pharmacokinetics and Biopharmacenics, 26, 689-716.

Tversky, Amos (1977), "Features of Similarity," Psychological Review, 84 (3), 327-352.

Verlegh, Peter W.J., Hendrik N.J. Schifferstein, and Dick R. Wittink (2002), "Range and Numberof-Levels Effects in Derived and Stated Measures of Attribute Importance," Marketing Letters, $13(1), 41-52$.

Wittink, Dick R., Joel Huber, Peter Zandan, and Richard M. Johnson (1992), "The Number of Levels Effect in Conjoint: Where Does It Come From, And Can It Be Eliminated?" in Sawtooth Software Conference Proceedings.

Wittink, Dick R., Lakshman Krishnamurthi, and Julia B. Nutter (1982), "Comparing Derived Importance Weights Across Attributes," Journal of Consumer Research, 8, 471-474. 
Wittink, Dick R., Lakshman Krishnamurthi, and David J. Reibstein (1990), "The Effect of Differences in the Number of Attribute Levels on Conjoint Analysis," Marketing Letters, 1 (2), 113-123.

Wittink, Dick R., William G. McLauchlan, and P.B. Seetharaman (1997), "Solving the Number-ofAttribute-Levels Problem in Conjoint Analysis," in Sawtooth Software Conference proceedings. 